\title{
Existence of Solutions of Multi-Point BVPs for Impulsive Functional Differential Equations with Nonlinear Boundary Conditions
}

Yuji Liu

Department of Mathematics, Guangdong University of Business Studies, Guangzhou, P R China.

This paper is dedicated to Professor Ljubomir Ćirić

Communicated by Professor V. Berinde

\begin{abstract}
Two classes of multi-point BVPs for first order impulsive functional differential equations with nonlinear boundary conditions are studied. Sufficient conditions for the existence of at least one solution to these BVPs are established, respectively. Our results generalize and improve the known ones. Some examples are presented to illustrate the main results.(C)2012 NGA. All rights reserved.
\end{abstract}

Keywords: Nonlinear multi-point boundary value problem; first order impulsive functional differential equation; fixed-point theorem; growth condition.

2010 MSC: Primary 34B37; 65Q20; Secondary 65L05; 92D25.

\section{Introduction}

In recent years, there has been a large number of papers concerned with the solvability of periodic boundary value problems for first order [1-12,16,18,20,22-27,29-31], second order or higher order [13-16] impulsive functional differential equations. To illustrate the motivation of this paper and compare the results in this paper to known ones, we first present a survey on studies on boundary value problems for first order ordinary or functional differential equations with or without impulses effects.

${ }^{*}$ Corresponding author

Email address: liuyuji888@sohu.com (Yuji Liu) 
Jankowski in [17] studied the existence of solutions of boundary value problem for functional differential equation ( BVP for short)

$$
\left\{\begin{array}{l}
x^{\prime}(t)=f(t, x(t), x(\alpha(t))) \equiv F x(t), t \in[0, T], T>0 \\
x(0)=\lambda x(T)+k
\end{array}\right.
$$

where $f$ is continuous, $\alpha:[0, T] \rightarrow[0, T]$ continuous, $\lambda, k \in R$. Using Banach's fixed point theorem, it was proved that $\mathrm{BVP}(1.1)$ has unique solutions under some assumptions, one of which is as follows:

$\left(M_{1}\right)$. It holds that

$$
\left|f\left(t, u_{1}, u_{2}\right)+M u_{1}-f\left(t, v_{1}, v_{2}\right)-M v_{1}\right| \leq K_{1}\left|u_{1}-v_{1}\right|+K_{2}\left|u_{2}-v_{2}\right|, t \in[0, T]
$$

when $u_{1}, u_{2}, v_{1}, v_{2} \in R$ for case $\lambda>0$ or

$$
\left|f\left(t, u_{1}, u_{2}\right)-f\left(t, v_{1}, v_{2}\right)\right| \leq K_{1}\left|u_{1}-v_{1}\right|+K_{2}\left|u_{2}-v_{2}\right|, t \in[0, T]
$$

when $u_{1}, u_{2}, v_{1}, v_{2} \in R$ for case $\lambda<0$.

By applying upper and lower solutions methods and monotone iterative technique, it was proved in [17] that BVP(1.1) has extremal solutions under some conditions, one of the main assumptions is that the inequality

$$
f\left(t, u_{1}, u_{2}\right)-f\left(t, v_{1}, v_{2}\right) \leq K_{1}\left[u_{1}-v_{1}\right]+K_{2}\left[u_{2}-v_{2}\right]
$$

holds for $t \in[0, T], u_{1} \leq v_{1}, u_{2} \leq v_{2}$.

In paper [18], the authors investigated the following BVP with nonlinear boundary conditions

$$
\left\{\begin{array}{l}
x^{\prime}(t)=f(t, x(t)), t \in[0, T], T>0 \\
g(x(0), x(T))=0
\end{array}\right.
$$

where $f, g$ are continuous functions. The main assumptions in [7] are as follows.

$\left(M_{2}\right) . \alpha, \beta$ are sub-solution and super-solution of above problem respectively satisfying $\alpha(t) \leq \beta(t), t \in$ $[0, T]$

$\left(M_{3}\right) . \quad f$ and $g$ satisfy that

$$
f(t, v)+M v \leq f(t, u)+M u, t \in[0, T], \alpha(t) \leq v \leq u \leq \beta(t)
$$

and

$$
g\left(x^{\prime}, y\right)-m x^{\prime} \leq g(x, y)-m x, \quad g(x, y) \leq g\left(x, y^{\prime}\right)
$$

for $x, x^{\prime} \in[\alpha(0), \beta(0)]$ with $x \leq x^{\prime}$ and $y, y^{\prime} \in[\alpha(T), \beta(T)]$ with $y \leq y^{\prime}$;

$\left(M_{4}\right)$. there exist constants $m, m^{\prime}, m^{\prime \prime} \geq 0$ such that for every $x, x^{\prime} \in[\alpha(0), \beta(0)]$ and $y, y^{\prime} \in[\alpha(T), \beta(T)]$ with $x<x^{\prime}$ and $y<y^{\prime}$ the following growth conditions are satisfied

$$
-m^{\prime} \leq \frac{g\left(x^{\prime}, y\right)-g(x, y)}{x^{\prime}-x} \leq m, \quad 0 \leq \frac{g\left(x, y^{\prime}\right)-g(x, y)}{y^{\prime}-y} \leq m^{\prime \prime} .
$$

The author in recent paper [13] also studied the existence of solutions of BVP(1.2), but the methods used are different from those ones used in [18].

In paper [19], the author studied the existence of solutions of the BVP with nonlinear boundary conditions

$$
\left\{\begin{array}{l}
x^{\prime}(t)=f(t, x(t)), t \in[0, T], T>0 \\
g\left(x\left(t_{0}\right), x\left(t_{1}\right), \cdots, x\left(t_{r}\right)\right)=0
\end{array}\right.
$$

where $f, g$ are continuous functions, $0=t_{0}<t_{1}<\cdots<t_{r}=T$ fixed. The main assumptions in [19] are $\left(M_{2}\right),\left(M_{3}\right)$ mentioned above and 
$\left(M_{5}\right)$. there exists a constant $L>0$ such that

$$
g\left(x, u_{1}, \cdots, u_{r}\right)-g\left(y, u_{1}, \cdots, u_{r}\right) \leq L(x-y)
$$

for all $\alpha(0) \leq y \leq x \leq \beta(0)$ and $\alpha\left(t_{i}\right) \leq u_{i} \leq \beta\left(t_{i}\right), i=1, \cdots, r$.

Using fixed point theorems and the lower and upper solution methods, in [30], a pioneer paper concerning the solvability of periodic boundary value problem for first order impulsive differential equation ( IBVP for short ), Nieto studied the solvability of

$$
\left\{\begin{array}{l}
x^{\prime}(t)+\lambda x(t)=F(t, x(t)), \quad t \in[0, T] \backslash\left\{t_{1}, \cdots, t_{p}\right\} \\
x\left(t_{k}^{+}\right)-x\left(t_{k}\right)=I_{k}\left(x\left(t_{k}\right)\right), \quad k=1, \cdots, p \\
x(0=x(T),
\end{array}\right.
$$

where $\lambda \neq 0, J=[0, T], 0=t_{0}<t_{1}<\cdots<t_{p}<t_{p+1}=T$. Nieto transformed (4) into the following integral equation

$$
x(t)=\int_{0}^{T} g(t, s) F(s, x(s)) d s+\sum_{k=1}^{p} g\left(t, t_{k}\right) I_{k}\left(x\left(t_{k}\right)\right),
$$

where

$$
g(t, s)=\frac{1}{1-e^{-\lambda T}}\left\{\begin{array}{l}
e^{-\lambda(t-s)}, \quad 0 \leq s \leq t \leq T \\
e^{-\lambda(T+t-s)}, \quad 0 \leq t<s \leq T .
\end{array}\right.
$$

Then it was showed that $\operatorname{IBVP}(1.4)$ has at least one solution under one of the assumptions:

$\left(M_{6}\right) . \quad F$ is bounded and $I_{k}(k=1, \cdots, p)$ are bounded;

$\left(M_{7}\right)$. There is $l_{k}>0$ so that $\left|I_{k}(x)-I_{k}(y)\right| \leq l_{k}|x-y|$ and there is $l>0$ so that $|F(t, x)-F(t, y)| \leq l|x-y|$ hold for all $t \in J$ and $(x, y) \in R^{2}$;

$\left(M_{8}\right)$. There are $\alpha \in[0,1), \alpha_{k} \in[0,1)(k=1, \cdots, p)$ and $a_{k}, b_{k}, b \in R, a \in P C(J)$ so that

$$
|F(t, x)| \leq a(t)+b|x|^{\alpha},\left|I_{k}(x)\right| \leq a_{k}+b_{k}|x|^{\alpha_{k}}, k=1, \cdots, p,
$$

hold for all $t \in J$ and $x \in R$.

In [20], Nieto considered the following IBVP with periodic boundary conditions

$$
\left\{\begin{array}{l}
x^{\prime}(t)+F(t, x(t))=0, \quad \text { a.e.t } \in[0,1] \backslash\left\{t_{1}, \cdots, t_{p}\right\} \\
x\left(t_{k}^{+}\right)-x\left(t_{k}\right)=I_{k}\left(x\left(t_{k}\right)\right), \quad k=1,2, \cdots, p \\
x(0)=x(T),
\end{array}\right.
$$

where $0=t_{0}<t_{1}<\cdots<t_{p}<t_{p+1}=1, F$ is an impulsive Carathéodory function, $I_{k}$ is continuous. Nieto proved the following theorem.

Theorem A[20]. Suppose there exist $r>0$ and $k>0$ such that

$$
\frac{F(t, u)}{u} \geq k>0 \text { a.e. } t \in J \text { and for every }|u| \geq r ; \lim _{u \rightarrow 0} \frac{I_{k}(u)}{u}=0 \text { for } k=1, \cdots, p .
$$

Then IBVP(1.5) has at least one solution.

In paper [21], the author proved that if there exist $r>0, k>0, c_{j}, k_{j} \in R$ and $\xi \in L^{1}(J)$ so that

$$
\begin{gathered}
\frac{F(t, u)}{u} \geq k+\frac{\xi(t)}{u}, \text { a.e. } t \in J,|u|>r, \\
\left|I_{k}(x)\right| \leq c_{k}+k_{k}|x|,|x|>r, k=1, \cdots, p,
\end{gathered}
$$




$$
\sum_{k=1}^{p} k_{j}<1-e^{-k T}
$$

then $\operatorname{IBVP}(1.5)$ has at least one solution.

In [22], Franco and Nieto studied the following IBVP

$$
\left\{\begin{array}{l}
x^{\prime}(t)=f(t, x(t)), \quad \text { a.e.t } \in J \backslash\left\{t_{1}, \cdots, t_{p}\right\} \\
x\left(t_{k}^{+}\right)-x\left(t_{k}\right)=I_{k}\left(x\left(t_{k}\right)\right), \quad k=1,2, \cdots, p \\
x(0)=x(T) .
\end{array}\right.
$$

Using upper and lower solutions method and the monotone technique, they proved IBVP(1.6) has at least one solution under the existence assumptions of lower solution $\alpha$ and upper solution $\beta$ and the following condition:

$\left(M_{9}\right) . I_{k}$ are continuous and nondecreasing and $f$ satisfies

$$
f(t, u)-f(t, v) \geq-M(u-v)
$$

for a.e. $t \in J$ and all $(u, v) \in R^{2}$ with $\alpha(t) \leq v \leq u \leq \beta(t)$, where $M=\min \left\{M_{\alpha}, M_{\beta}\right\}$ and $M_{\alpha}$ and $M_{\beta}$ satisfying

$$
-\int_{t_{p}}^{T} e^{-M_{\beta}(T-s)}\left[f(s, \beta(s))-\beta^{\prime}(s)\right] d s \geq \beta(T)-\beta(0)
$$

and

$$
\int_{t_{p}}^{T} e^{-M_{\alpha}(T-s)}\left[f(s, \alpha(s))-\alpha^{\prime}(s)\right] d s \geq \alpha(0)-\beta(T) .
$$

In a recent paper [23], Liu studied the following periodic boundary value problem of first order impulsive functional differential equation

$$
\left\{\begin{array}{l}
x^{\prime}(t)+a(t) x(t)=f\left(t, x(t), x\left(\alpha_{1}(t)\right), \cdots, x\left(\alpha_{n}(t)\right)\right), \text { a.e. } t \in[0, T], \\
x\left(t_{k}^{+}\right)-x\left(t_{k}\right)=I_{k}\left(x\left(t_{k}\right)\right), \quad k=1,2, \cdots, p \\
x(0)=x(T) .
\end{array}\right.
$$

Sufficient conditions for the existence of at least one solution of above mentioned IBVP were established in $[23]$.

In recent paper [24], Liu and Ge studied the existence of periodic solutions of the following first order differential equation with linear impulses effects

$$
\left\{\begin{array}{l}
x^{\prime}(t)+a(t) x(t)+F(t, x(t-\tau(t)))=0, \quad t \in R, t \neq t_{k}, k \in Z \\
x\left(t_{k}^{+}\right)-x\left(t_{k}\right)=b_{k} x\left(t_{k}\right), \quad k=1,2, \cdots
\end{array}\right.
$$

Using fixed point theorem, they proved that (1.7) has at least three positive periodic solutions under some assumptions imposed on $F$ and $b_{k}$, and at least one periodic solution under some other assumption.

Recently, the authors in paper [11] studied the solvability of periodic boundary value problems for non-Lipschizian impulsive functional differential equations.

We find that, besides $[18,19]$, there was no other paper concerned with the existence of solutions of multi-point boundary value problems for first order impulsive differential equations with nonlinear boundary conditions.

In this paper, we investigate the existence of solutions of nonlinear multi-point boundary value problems for nonlinear first order impulsive functional differential equations with nonlinear boundary conditions

$$
\left\{\begin{array}{l}
x^{\prime}(t)=f\left(t, x(t), x\left(\alpha_{1}(t)\right), \cdots, x\left(\alpha_{n}(t)\right)\right), \text { a.e. } t \in[0, T], \\
\Delta x\left(t_{k}\right)=I_{k}\left(x\left(t_{1}\right), \cdots, x\left(t_{m}\right)\right), k=1, \cdots, m, \\
x(T)=g\left(x\left(s_{0}\right), x\left(s_{1}\right), \cdots, x\left(s_{r}\right)\right),
\end{array}\right.
$$


and

$$
\left\{\begin{array}{l}
x^{\prime}(t)=f\left(t, x(t), x\left(\alpha_{1}(t)\right), \cdots, x\left(\alpha_{n}(t)\right)\right), \text { a.e. } t \in[0, T], \\
\Delta x\left(t_{k}\right)=I_{k}\left(x\left(t_{1}\right), \cdots, x\left(t_{m}\right)\right), k=1, \cdots, m, \\
x(0)=g\left(x\left(s_{0}\right), x\left(s_{1}\right), \cdots, x\left(s_{r}\right)\right),
\end{array}\right.
$$

where $T>0,0=s_{0}<s_{1}<\cdots<s_{r}=T$ and $0<t_{1}<\cdots<t_{m}<T$ are constants, $\alpha_{k} \in C^{1}([0, T],[0, T])$ for all $k=1, \cdots, n$, and its inverse function denoted by $\beta_{k}, f$ is an impulsive Carathéodory function, $I_{k}$ and $g$ are continuous functions, $\Delta x\left(t_{k}\right)=x\left(t_{k}^{+}\right)-x\left(t_{k}^{-}\right)$. New results on the existence of solutions of $\operatorname{IBVP}(1.8)$ and IBVP(1.9) are established, respectively. The technical methods used are motivated by [23] and are different from those in $[2,18,16,19,25,9,26,21,27]$.

Applying the main results obtained to the following BVPs with impulses effects

$$
\left\{\begin{array}{l}
x^{\prime}(t)=f(t, x(t), x(\alpha(t))) \equiv F x(t), t \in[0, T], T>0, \\
\Delta x\left(t_{k}\right)=I_{k}\left(x\left(t_{k}\right)\right), k=1, \cdots, m \\
x(0)=\lambda x(T)+k
\end{array}\right.
$$

and

$$
\left\{\begin{array}{l}
x^{\prime}(t)=f(t, x(t)), t \in[0, T], T>0, \\
\Delta x\left(t_{k}\right)=I_{k}\left(x\left(t_{k}\right)\right), k=1, \cdots, m, \\
g\left(x\left(s_{0}\right), x\left(s_{1}\right), \cdots, x\left(s_{r}\right)\right)=0
\end{array}\right.
$$

where $0<t_{1}<\cdots<t_{p}<T$ and $I_{k}$ is continuous for $k=1, \cdots, p, f$ is continuous, $\alpha:[0, T] \rightarrow[0, T]$ continuous, $\lambda, k \in R, f, g, I_{k}$ are continuous functions, $0=s_{0}<s_{1}<\cdots<s_{r}=T$ and $0<t_{1}<\cdots<t_{m}<T$ fixed, the corollaries are novelty, generalize those ones in [17] and the methods used are different from those ones in $[12,14,17]$.

The remainder of this paper is divided into two sections. In Section 2, we present the main results ( Theorem 2.1 and Theorem 2.2 ), and some examples to illustrate the theorems are also given in this section. In Section 3, we prove Theorem 2.1 and Theorem 2.2.

\section{Main Results and Examples}

In this section, we establish the main results. To define solutions of IBVP(1.8) or IBVP(1.9), we first introduce two Banach spaces.

Let $u: J=[0, T] \rightarrow R$, and $0=t_{0}<t_{1}<\cdots<t_{m}<t_{m+1}=T$, for $k=0, \cdots, m$, define the function $u_{k}:\left(t_{k}, t_{k+1}\right) \rightarrow R$ by $u_{k}(t)=u(t)$. We will use the following sets

$$
X=\left\{\begin{array}{l}
x: J \rightarrow R, x_{k} \in C^{0}\left(t_{k}, t_{k+1}\right), k=0, \cdots, m, \\
\text { there exist the limits }\left\{\begin{array}{l}
\lim _{t \rightarrow t_{k}^{-}} x(t)=x\left(t_{k}\right), \\
\lim _{t \rightarrow t_{k}^{+}} x(t), \\
\lim _{t \rightarrow 0^{+}} x(t)=x(0), \\
\lim _{t \rightarrow T^{-}} x(t)=x(T)
\end{array}\right\}
\end{array}\right.
$$

and

$$
Y=X \times R^{m+1}
$$

with the norms

$$
\|u\|=\|u\|_{X}=\max \left\{\sup _{t \in\left(t_{k}, t_{k+1}\right)}\left|u_{k}(t)\right|, k=0, \cdots, m\right\}
$$

for $u \in X$ and

$$
\|y\|=\|y\|_{Y}=\max \left\{\|u\|_{X}, \max _{1 \leq k \leq m+1}\left\{\left|x_{k}\right|\right\}\right\}
$$


for $y=\left\{u, x_{1}, \cdots, x_{m+1}\right\} \in Y$, respectively. It is easy to show that $X$ and $Y$ are Banach spaces.

A function $F$ is called an impulsive Carathéodory function if

$* F\left(\bullet, u_{0}, u_{1}, \cdots, u_{n}\right) \in X$ for each $u=\left(u_{0}, u_{1}, \cdots, u_{n}\right) \in R^{n}$;

$* F(t, \bullet, \cdots, \bullet)$ is continuous for a.e. $t \in J \backslash\left\{t_{1}, \cdots, t_{m}\right\}$;

* for each $r>0$ there exists $h_{r} \in L^{1}(J)$ such that

$$
\left|F\left(t, u_{0}, u_{1}, \cdots, u_{n}\right)\right| \leq h_{r}(t)
$$

holds for a.e. $t \in J \backslash\left\{t_{1}, \cdots, t_{m}\right\}$ and every $u$ satisfying $\max _{i=0,1, \cdots, n}\left|u_{i}\right| \leq r$.

By a solution of $\operatorname{IBVP}(1.8)$ ( or IBVP(1.9) ) we mean a function $u \in X$ satisfying all equations in (1.8) (or (1.9)).

The main results are as follows:

Theorem 2.1. Suppose

(A) there exists a constant $M>0$ such that $I_{k}\left(x_{1}, \cdots, x_{m}\right) x_{k} \geq-\frac{M}{m}$ for all $x_{1}, \cdots, x_{m} \in R$ and $k=1, \cdots, m$;

(C) there exist functions $h:[0, T] \times R^{n+1} \rightarrow R, g_{i}:[0, T] \times R \rightarrow R(i=0,1, \cdots, n)$ and $r:[0, T] \rightarrow R$ such that

(i) $f\left(t, x_{0}, \cdots, x_{n}\right)=h\left(t, x_{0}, \cdots, x_{n}\right)+\sum_{i=0}^{n} g_{i}\left(t, x_{i}\right)+r(t)$ holds for all $\left(t, x_{0}, \cdots, x_{n}\right) \in[0, T] \times R^{n+1}$;

(ii) $g_{i}(t, x)(i=0,1,2,3, \cdots, n)$ satisfies that $g_{i}(\bullet, x) \in X$ for every $x \in R$ and $g_{i}(t, \bullet)$ is continuous for a.e. $t \in[0, T], r \in X$;

(iii) $h$ satisfies that $h\left(\bullet, x_{0}, \cdots, x_{n}\right) \in X$ for every $\left(x_{0}, \cdots, x_{n}\right) \in R^{n+1}$ and $h(t, \bullet, \cdots, \bullet)$ is continuous for a.e. $t \in[0, T]$;

(iv) There exist constants $\theta \geq 0$ and $\beta>0$ such that

$$
h\left(t, x_{0}, \cdots, x_{n}\right) x_{0} \geq \beta\left|x_{0}\right|^{\theta+1}
$$

holds for all $\left(t, x_{0}, \cdots, x_{n}\right) \in[0, T] \times R^{n+1}$;

(v) $\lim _{|x| \rightarrow+\infty} \sup _{t \in[0, T]} \frac{\left|g_{i}(t, x)\right|}{|x|^{\theta}}=r_{i} \in[0,+\infty)$ for $i=0,1,2, \cdots, n$, where $\theta$ is defined in (iv);

(D) for each $\delta>0, \max _{\left|x_{0}\right| \leq \delta}\left|g\left(x_{0}, \cdots, x_{r}\right)\right|$ is bounded and

$$
\lim _{x_{0} \rightarrow \infty} \frac{\left|g\left(x_{0}, x_{1}, \cdots, x_{r}\right)\right|}{\left|x_{0}\right|}=\alpha<1 \text { uniformly in }\left(x_{1}, \cdots, x_{r}\right) \in R^{r} .
$$

Then IBVP(1.8) has at least one solution if

$$
r_{0}+\sum_{k=1}^{n} r_{k}\left\|\beta_{k}^{\prime}\right\|^{\theta /(1+\theta)}<\beta .
$$

Theorem 2.2. Suppose

(A1) there exists a constant $M>0$ such that $\left(2 x_{k}+I_{k}\left(x_{1}, \cdots, x_{m}\right)\right) I_{k}\left(x_{1}, \cdots, x_{m}\right) \leq \frac{M}{m}$ for all $x_{1}, \cdots, x_{m} \in R$ and $k=1, \cdots, m$;

(C1) there exist functions $h:[0, T] \times R^{n+1} \rightarrow R, g_{i}:[0, T] \times R \rightarrow R(i=0,1, \cdots, n)$ and $r:[0, T] \rightarrow R$ such that $(\mathbf{C})(\mathbf{i}),(\mathbf{C})(\mathbf{i i}),(\mathbf{C})(\mathbf{i i i})$ and $(\mathbf{C})(\mathbf{v})$ in Theorem 2.1 hold and

(iv) there exist constants $\theta \geq 0$ and $\beta>0$ such that

$$
h\left(t, x_{0}, \cdots, x_{n}\right) x_{0} \leq-\beta\left|x_{0}\right|^{\theta+1}
$$

holds for all $\left(t, x_{0}, \cdots, x_{n}\right) \in[0, T] \times R^{n+1}$; 
(D1) for each $\delta>0, \max _{\left|x_{r}\right| \leq \delta}\left|g\left(x_{0}, \cdots, x_{r}\right)\right|$ is bounded and

$$
\lim _{x_{r} \rightarrow \infty} \frac{\left|g\left(x_{0}, x_{1}, \cdots, x_{r}\right)\right|}{\left|x_{r}\right|}=\alpha<1 \text { uniformly in }\left(x_{0}, \cdots, x_{r-1}\right) \in R^{r}
$$

Then IBVP(1.9) has at least one solution if (2.1) holds.

Corollary 2.1. Suppose

(A0) $I_{k}\left(x_{1}, \cdots, x_{m}\right) x_{k} \geq-M$ for all $x_{1}, \cdots, x_{m} \in R$ and $k=1, \cdots, m$;

and (C), (D) in Theorem 2.1 hold. Then IBVP(1.8) has at least one solution if (10) hold.

Corollary 2.2. Suppose

(A10) $\left(2 x_{k}+I_{k}\left(x_{1}, \cdots, x_{m}\right)\right) I_{k}\left(x_{1}, \cdots, x_{m}\right) \leq M$ for all $x_{1}, \cdots, x_{m} \in R$ and $k=1, \cdots, m$;

and (C1), (D1) in Theorem 2.2 hold. Then IBVP(1.9) has at least one solution if (2.1) hold.

Now, we present some examples to illustrate above theorems. Since the boundary conditions in examples are non-homogeneous, these examples can not be solved by the results in known papers $[1,13,14,16,17,34,10-$ $12,5,28-32]$ and [23].

Example 2.1. Consider the following IBVP

$$
\left\{\begin{array}{l}
x^{\prime}(t)=\sum_{k=1}^{2 p+1} a_{k} x^{k}(t)+r(t), \\
\Delta x\left(t_{k}\right)=b_{k}\left[x\left(t_{k}\right)\right]^{\alpha}, k=1, \cdots, m, \\
x(T)=\lambda x(0)+k
\end{array} \quad t \in[0, T], t \neq t_{k}, k=1, \cdots, m,\right.
$$

where $p$ a nonnegative integer, $m$ a positive integer, $\alpha$ is a ratio of two positive odd integers, $T>0$, $0<t_{1}<\cdots<t_{m}<T, s_{0}=0, s_{1}=T, b_{k} \in R$ for all $k=1, \cdots, m, a_{2 p+1} \in R$ and $a_{k} \in R$ for all $k=1, \cdots, 2 p, r \in X, \lambda \in R, k \in R$.

Case 1. $|\lambda|<1$.

Proof. Corresponding to $\operatorname{IBVP}(1.8)$, one sees that

$$
\begin{aligned}
& f\left(t, x_{0}\right)=\sum_{k=0}^{2 p+1} a_{k} x_{0}^{k}+r(t), \\
& I_{k}\left(x_{1}, \cdots, x_{m}\right)=b_{k} x_{k}^{\alpha}, \quad k=1, \cdots, m, \\
& g\left(x_{0}, x_{1}\right)=\lambda x_{0}+k .
\end{aligned}
$$

It is easy to see that

(A). since $\alpha$ is a ratio of two odd positive integers, we have $I_{k}\left(x_{1}, \cdots, x_{m}\right) x_{k}=b_{k}\left[x_{k}\right]^{\alpha+1} \geq 0$ for all $x_{1}, \cdots, x_{m} \in R$ and $k=1, \cdots, m$ if $b_{k} \geq 0(i=1,2, \cdots, m)$.

(C). Let $h\left(t, x_{0}\right)=a_{2 p+1} x_{0}^{2 p+1}, g_{0}\left(t, x_{0}\right)=\sum_{k=1}^{2 p} a_{k} x_{0}^{k}$; Then (C)(i),(C)(ii),(C)(iii) in Theorem 2.1 hold. Furthermore, (C)(iv) in Theorem 2.1 holds with $\beta=a_{2 p+1}>0$ and $\theta=2 p+1$ if $a_{2 p+1}>0$; (C)(v) holds with $r_{0}=0$.

(D). $\lim _{\left|x_{0}\right| \rightarrow+\infty} \frac{\left|g\left(x_{0}, x_{1}\right)\right|}{\left|x_{0}\right|}=\alpha=|\lambda|<1$.

One sees that $(2.1)$ holds since $r_{0}=0$. It follows from Corollary 2.1 that $\operatorname{IBVP}(2.2)$ has at least one solution if $a_{2 p+1}>0$ and $b_{k} \geq 0(k=1,2, \cdots, m)$.

Case 2. $|\lambda|>1$.

At this case, we have $1 /|\lambda|<1$. Transform $\operatorname{IBVP}(2.2)$ into

$$
\left\{\begin{array}{l}
x^{\prime}(t)=\sum_{k=1}^{2 p+1} a_{k} x^{k}(t)+r(t), t \in[0, T], t \neq t_{k}, k=1, \cdots, m \\
\Delta x\left(t_{k}\right)=b_{k}\left[x\left(t_{k}\right)\right]^{\alpha}, k=1, \cdots, m \\
x(0)=\frac{1}{\lambda} x(T)-\frac{k}{\lambda}
\end{array}\right.
$$


Corresponding to $\operatorname{IBVP}(1.9)$, one sees that

$$
\begin{aligned}
& f\left(t, x_{0}\right)=\sum_{k=0}^{2 p+1} a_{k} x_{0}^{k}+r(t), \\
& I_{k}\left(x_{1}, \cdots, x_{m}\right)=b_{k}\left[x_{k}\right]^{\alpha}, \quad k=1, \cdots, m, \\
& g\left(x_{0}, x_{1}\right)=\frac{1}{\lambda} x_{1}-\frac{k}{\lambda} .
\end{aligned}
$$

It is easy to see that

(A1). $\left[2 x_{k}+I_{k}\left(x_{1}, \cdots, x_{p}\right)\right] I_{k}\left(x_{1}, \cdots, x_{p}\right)=\left[2 x_{k}+b_{k}\left[x_{k}\right]^{\alpha}\right] b_{k}\left[x_{k}\right]^{\alpha} \leq 0$ for all $x_{1}, \cdots, x_{m} \in R$ and $k=1, \cdots, m$ if $\alpha=1$ and $\left(2+b_{k}\right) b_{k} \leq 0$.

(C1). Let $h\left(t, x_{0}\right)=a_{2 p+1} x_{0}^{2 p+1}, g_{0}\left(t, x_{0}\right)=\sum_{k=1}^{2 p} a_{k} x_{0}^{k}$. Then (C)(i),(C)(ii),(C)(iii) in Theorem 2.1 hold; (C1)(iv) holds with $\theta=2 p+1$ and $\beta=a_{2 p+1}$ if $a_{2 p+1}<0$; (C)(v) holds with $r_{0}=0$.

(D1). $\lim _{\left|x_{1}\right| \rightarrow+\infty} \frac{\left|g\left(x_{0}, x_{1}\right)\right|}{\left|x_{1}\right|}=\alpha=\frac{1}{|\lambda|}<1$.

It follows from Corollary $\mathbf{2 . 2}$ that $\operatorname{IBVP}(2.2)$ has at least one solution if $\alpha=1, a_{2 p+1}<0$, and $b_{k}\left(2+b_{k}\right) \leq 0$ for all $k=1,2, \cdots, m$.

Case 3. $|\lambda|=1$.

Let $y(t)=e^{-t} x(t)$, then $x^{\prime}(t)=e^{t}\left[y(t)+y^{\prime}(t)\right]$ and

$$
\Delta y\left(t_{k}\right)=y\left(t_{k}^{+}\right)-y\left(t_{k}\right)=e^{-t_{k}} x\left(t_{k}^{+}\right)-e^{t_{k}} x\left(t_{k}\right)=e^{t_{k}} \Delta x\left(t_{k}\right)=b_{k} e^{-t_{k}}\left[x\left(t_{k}\right)\right]^{\alpha}=b_{k} e^{(\alpha-1) t_{k}} y\left(t_{k}\right) .
$$

We change $\operatorname{IBVP}(2.2)$ to

$$
\left\{\begin{array}{l}
y^{\prime}(t)=-y(t)+\sum_{k=1}^{2 p+1} a_{k} e^{(k-1) t} y^{k}(t)+r(t) e^{-t}, t \in[0, T], t \neq t_{k}, k=1, \cdots, m, \\
\Delta y\left(t_{k}\right)=b_{k} e^{(\alpha-1) t_{k}}\left[y\left(t_{k}\right)\right]^{\alpha}, k=1, \cdots, m \\
y(T)=\frac{\lambda}{e^{T}} y(0)+\frac{k}{e^{T}}
\end{array}\right.
$$

Corresponding to $\operatorname{IBVP}(1.8)$, one sees that

$$
\begin{aligned}
& f\left(t, x_{0}\right)=-x_{0}+\sum_{k=1}^{2 p+1} a_{k} e^{(k-1) t} x_{0}^{k}+r(t), \\
& I_{k}\left(x_{1}, \cdots, x_{m}\right)=b_{k} e^{(\alpha-1) t_{k}}\left[x_{k}\right]^{\alpha}, \quad k=1, \cdots, m, \\
& g\left(x_{0}, x_{1}\right)=\frac{\lambda}{e^{T}} x_{0}+\frac{k}{e^{T}} .
\end{aligned}
$$

It is easy to see that

(A). since $\alpha$ is a ratio of two odd positive integers, we have $I_{k}\left(x_{1}, \cdots, x_{p}\right) x_{k}=b_{k} e^{(\alpha-1) t_{k}}\left[x_{k}\right]^{\alpha+1} \geq 0$ for all $x_{1}, \cdots, x_{m} \in R$ and $k=1, \cdots, m$ if $b_{k} \geq 0$ for all $k=1,2, \cdots, m$;

(C). Let $h\left(t, x_{0}\right)=a_{2 p+1} x_{0}^{2 p+1}, g_{0}\left(t, x_{0}\right)=-x_{0}+\sum_{k=1}^{2 p} a_{k} x_{0}^{k}, r(t)$ be replaced by $r(t) e^{-t}$. Then (C)(i), (C) (ii), (C)(iii) hold; (C)(iv) holds with $\theta=2 p+1$ and $\beta=a_{2 p+1}$ if $a_{2 p+1}>0$; (C)(v) holds with $r_{0}=0$ if $p>0$.

(D). $\lim _{\left|x_{0}\right| \rightarrow+\infty} \frac{\left|g\left(x_{0}, x_{1}\right)\right|}{\left|x_{0}\right|}=\alpha=\frac{1}{e^{T}}<1$.

One sees that (2.1) holds since $r_{0}=0$. Then Corollary 2.1 implies that $\operatorname{IBVP}(2.2)$ has at least one solution if $p>0, a_{2 p+1}>0$ and $b_{k} \geq 0$ for all $k=1,2, \cdots, m$.

If $p=0$, one sees that (A) and (D) in Theorem 2.1 hold and

(C). Let $h\left(t, x_{0}\right)=\left(a_{1}-1\right) x_{0}, g_{0}\left(t, x_{0}\right)=0, r(t)$ be replaced by $r(t) e^{-t}$. Then (C)(i), (C) (ii), (C)(iii) hold; (C)(iv) holds with $\theta=1$ and $\beta=a_{1}-1$ if $a_{1}-1>0 ;(\mathbf{C})(\mathbf{v})$ holds with $r_{0}=0$.

One sees that (2.1) holds since $r_{0}=0$. Hence Corollary 2.1 implies that $\operatorname{IBVP}(2.2)$ has at least one solution if $a_{2 p+1}>1$ and $b_{k} \geq 0$ for all $k=1,2, \cdots, m$. 
Remark. Consider the following BVP

$$
\left\{\begin{array}{l}
x^{\prime}(t)=\sum_{k=1}^{2 p+1} a_{k} x^{k}(t)+r(t), t \in[0, T], t \neq t_{k}, k=1, \cdots, m, \\
\Delta x\left(t_{k}\right)=b_{k} x\left(t_{k}\right)+c_{k}, k=1, \cdots, m \\
x(T)=\lambda x(0)+k
\end{array}\right.
$$

where $p$ a nonnegative integer, $m$ a positive integer, $T>0,0<t_{1}<\cdots<t_{m}<T, s_{0}=0, s_{1}=T, b_{k} \geq 0$ for all $k=1, \cdots, m, c_{k} \in R$ for all $k=1, \cdots, m, a_{2 p+1} \in R$ and $a_{k} \in R$ for all $k=1, \cdots, 2 p, r \in X$, $\lambda \in R, k \in R$.

It is easy to see that

$$
x_{k} I_{k}\left(x_{1}, x_{2}, \cdots, x_{m}\right)=b_{k} x_{k}^{2}+c_{k} x_{k}=b_{k}\left(x_{k}+\frac{c_{k}}{2 b_{k}}\right)^{2}-\frac{c_{k}^{2}}{2 b_{k}} \geq-\frac{c_{k}^{2}}{2 b_{k}} .
$$

Hence (A) in Theorem 2.1 holds. Similarly to above discussion, we can get the existence results of this BVP by using Theorem 2.1.

Example 2.2. Consider the following IBVP

$$
\left\{\begin{array}{c}
x^{\prime}(t)=a_{2 p+1}\left(1+x^{2}(t)+\sum_{k=1}^{2 n+1} x^{2}\left(\frac{1}{k} t\right)\right) x^{2 p+1}(t)+\sum_{k=1}^{2 p} a_{k} x^{k}(t) \\
+\sum_{k=1}^{2 n+1} c_{k} x^{2 p+1}\left(\frac{1}{k} t\right)+r(t) \\
\quad t \in[0, T], t \neq t_{k}, k=1, \cdots, m \\
\begin{array}{c}
\Delta x\left(t_{k}\right)=b_{k}\left[x\left(t_{k}\right)\right]^{3}, k=1, \cdots, m \\
x(T)=\frac{1}{2}[x(0)]^{\alpha}+a \sin x(\xi)+b
\end{array}
\end{array}\right.
$$

where $T>0, p$ is a positive integer, $a_{2 p+1}>0, c_{2 m+1} \in R$, and $a_{k}, c_{k} \in R$ for all $k=1, \cdots, 2 p, r \in X$, $0<t_{1}<\cdots<t_{m}<T, b_{k} \geq 0$ for all $k=1, \cdots, m, 0 \leq \alpha \leq 1, \xi \in(0, T), a, b \in R$.

Proof. Corresponding to $\operatorname{IBVP}(1.8)$, one sees that $s_{0}=0, s_{1}=\xi, s_{2}=T$ and

$$
\begin{aligned}
& f\left(t, x_{0}, \cdots, x_{2 n+1}\right)=a_{2 p+1}\left(1+\sum_{i=0}^{2 n+1} x_{i}^{2}\right) x_{0}^{2 p+1}+\sum_{k=1}^{2 p} a_{k} x_{0}^{k}+\sum_{k=1}^{2 n+1} c_{k} x_{k}^{2 p+1}+r(t), \\
& I_{k}\left(x_{1}, \cdots, x_{m}\right)=b_{k}\left[x_{k}\right]^{3}, \quad k=1, \cdots, p, \\
& g\left(x_{0}, x_{1}, x_{2}\right)=\frac{1}{2}\left[x_{0}\right]^{\alpha}+a \sin x_{1}+b, \\
& \alpha_{k}(t)=\frac{1}{k} t, k=1, \cdots, 2 n+1 .
\end{aligned}
$$

It is easy to see that $\beta_{k}(t)=k t$ with $\left\|\beta_{k}\right\|=k T$ and

(A). $I_{k}\left(x_{1}, \cdots, x_{p}\right) x_{k}=b_{k}\left[x_{k}\right]^{4} \geq 0$ for all $x_{1}, \cdots, x_{m} \in R$ and $k=1, \cdots, m$ since $b_{k} \geq 0$.

(C). Let

$$
\begin{aligned}
& h\left(t, x_{0}, \cdots, x_{2 n+1}\right)=a_{2 p+1}\left(1+\sum_{i=0}^{2 n+1} x_{i}^{2}\right) x_{0}^{2 p+1}, \\
& g_{0}\left(t, x_{0}\right)=\sum_{k=1}^{2 p} a_{k} x_{0}^{k}, \\
& g_{i}\left(t, x_{i}\right)=c_{i} x_{i}^{2 p+1}(i=1, \cdots, 2 n+1),
\end{aligned}
$$

and $r$ be defined in $\operatorname{IBVP}(12)$. Then (C)(i), (C)(ii), (C)(iii) hold; (C)(iv) holds with $\theta=2 p+1$ and $\beta=a_{2 p+1}$ if $a_{2 p+1}>0 ;(\mathbf{C})(\mathbf{v})$ holds with $r_{0}=0$ and $r_{i}=\left|c_{i}\right|(i=1, \cdots, 2 n+1)$.

(D). $\lim _{\left|x_{0}\right| \rightarrow+\infty} \frac{\left|g\left(x_{0}, x_{1}, x_{2}\right)\right|}{\left|x_{0}\right|}= \begin{cases}0, & \alpha \in[0,1), \quad<1 . \\ \frac{1}{2}, & \alpha=1\end{cases}$ 
It follows from Corollary 2.1 that $\operatorname{IBVP}(2.3)$ has at least one solution if

$$
T^{\frac{2 p+1}{2 p+2}} \sum_{k=1}^{2 p+1} k^{\frac{2 p+1}{2 p+2}}\left|c_{k}\right|<a_{2 p+1} .
$$

\section{Proofs of Theorems}

In this section, we prove theorems given in Section 2. The following abstract existence theorem will be used, whose proof can be see in [7].

Lemma 3.1. Let $X$ and $Y$ be Banach spaces. Suppose $L: D(L) \subset X \rightarrow Y$ is a Fredholm operator of index zero with $\operatorname{Ker} L=\{0\}, N: X \rightarrow Y$ is $L$-compact on any open bounded subset of $X$. If $0 \in \Omega \subset X$ is an open bounded subset and $L x \neq \lambda N x$ for all $x \in D(L) \cap \partial \Omega$ and $\lambda \in[0,1]$, then there exist at least one $x \in \Omega$ such that $L x=N x$.

Consider $\operatorname{IBVP}(8)$, we define the linear operator $L: \operatorname{Dom} L \subseteq X \rightarrow Y$ and the nonlinear operator $N: X \rightarrow Y$ by

$$
L x(t)=\left(\begin{array}{c}
x^{\prime}(t) \\
\Delta x\left(t_{1}\right) \\
\cdot \\
\cdot \\
\cdot \\
\Delta x\left(t_{m}\right) \\
x(T)
\end{array}\right) \text { for } x \in D(L)
$$

where $D(L)=\left\{u \in X, u_{k} \in C^{1}\left(t_{k}, t_{k+1}\right), k=0,1, \cdots, m\right\}$ and

$$
N x(t)=\left(\begin{array}{c}
f\left(t, x(t), x\left(\alpha_{1}(t)\right), \cdots, x\left(\alpha_{n}(t)\right)\right) \\
I_{1}\left(x\left(t_{1}\right), \cdots, x\left(t_{m}\right)\right) \\
\cdot \\
\cdot \\
I_{m}\left(x\left(t_{1}\right), \cdots, x\left(t_{m}\right)\right) \\
g\left(x\left(s_{0}\right), x\left(s_{1}\right), \cdots, x\left(s_{r}\right)\right)
\end{array}\right) \text { for } x \in X
$$

Since

$$
\left\{\begin{array}{c}
x^{\prime}(t)=0 \\
\Delta x\left(t_{1}\right)=0 \\
\cdot \\
\cdot \\
\Delta x\left(t_{m}\right)=0 \\
x(T)=0
\end{array}\right.
$$

has unique solution $x(t) \equiv 0$, and $I_{k}, g$ are continuous, $f$ is Carathéodory function, we have the followings

(i). $\operatorname{Ker} L=\{0\}$.

(ii). $L$ is a Fredholm operator of index zero.

(iii). Let $\Omega \subset X$ be an open bounded subset with $\bar{\Omega} \cap D(L) \neq \emptyset$, then $N$ is $L$-compact on $\bar{\Omega}$.

(iv). $x \in D(L)$ is a solution of $B V P(8)$ if and only if $x$ is a solution of the operator equation $L x=N x$ in $D(L)$. 
Proof of Theorem 2.1. Let $\lambda \in(0,1)$. Suppose $x$ is a solution of the system

$$
\left\{\begin{array}{l}
x^{\prime}(t)=\lambda f\left(t, x(t), x\left(\alpha_{1}(t)\right), \cdots, x\left(\alpha_{n}(t)\right)\right), \text { a.e. } t \in[0, T], \\
\Delta x\left(t_{k}\right)=\lambda I_{k}\left(x\left(t_{1}\right), \cdots, x\left(t_{m}\right)\right), k=1, \cdots, m, \\
x(T)=\lambda g\left(x\left(s_{0}\right), x\left(s_{1}\right), \cdots, x\left(s_{r}\right)\right) .
\end{array}\right.
$$

We divide the remainder of the proof into two steps.

Step 1. Prove that there exists $\xi \in[0, T]$ and a constant $M^{\prime}>0$ such that $|x(\xi)| \leq M^{\prime}$.

Since (D) holds, we get that there exist constants $\delta^{\prime}>0$ and $\alpha_{1} \in[\alpha, 1)$ such that

$$
\frac{\left|g\left(x_{0}, x_{1}, \cdots, x_{r}\right)\right|}{\left|x_{0}\right|}<\alpha_{1} \text { for all }\left|x_{0}\right|>\delta^{\prime} \text { and }\left(x_{1}, \cdots, x_{r}\right) \in R^{r} .
$$

If $\left|x\left(s_{0}\right)\right|=|x(0)| \leq \delta^{\prime}$, then this Step is completed with $\xi=0$ and $M^{\prime}=\delta^{\prime}$. If $|x(0)|>\delta^{\prime}$, then we do the following.

Multiplying two sides of the first equation in (3.1) by $x(t)$, integrating it from 0 to $T$, we get from (C)(i) that

$$
\begin{aligned}
& \frac{1}{2}(x(T))^{2}-\frac{1}{2}(x(0))^{2}-\frac{1}{2} \sum_{k=1}^{m}\left[\left(x\left(t_{k}^{+}\right)\right)^{2}-\left(x\left(t_{k}^{-}\right)\right)^{2}\right] \\
= & \lambda \int_{0}^{T} f\left(s, x(s), x\left(\alpha_{1}(s)\right), \cdots, x\left(\alpha_{n}(s)\right)\right) x(s) d s \\
= & \lambda\left(\int_{0}^{T} h\left(s, x(s), x\left(\alpha_{1}(s)\right), \cdots, x\left(\alpha_{n}(s)\right)\right) x(s) d s+\int_{0}^{T} g_{0}(s, x(s)) x(s) d s\right. \\
& +\sum_{i=1}^{n} \int_{0}^{T} g_{i}\left(s, x\left(\alpha_{i}(s)\right) x(s) d s+\int_{0}^{T} r(s) x(s) d s\right) .
\end{aligned}
$$

It follows from (A) that

$$
\begin{aligned}
& \left(x\left(t_{k}^{+}\right)\right)^{2}-\left(x\left(t_{k}^{-}\right)\right)^{2}=\left(x\left(t_{k}^{+}\right)-x\left(t_{k}^{-}\right)\right)\left(x\left(t_{k}^{+}\right)+x\left(t_{k}^{-}\right)\right) \\
= & \Delta x\left(t_{k}\right)\left(2 x\left(t_{k}\right)+\Delta x\left(t_{k}\right)\right) \\
= & \lambda I_{k}\left(x\left(t_{1}\right), \cdots, x\left(t_{m}\right)\right)\left(2 x\left(t_{k}\right)+\lambda I_{k}\left(x\left(t_{1}\right), \cdots, x\left(t_{m}\right)\right)\right) \\
\geq & 2 \lambda x\left(t_{k}\right) I_{k}\left(x\left(t_{1}\right), \cdots, x\left(t_{m}\right)\right) \geq-2 \lambda \frac{M}{m} .
\end{aligned}
$$

Since

$$
\begin{aligned}
x(T)^{2}-x(0)^{2} & =\lambda^{2} g\left(x\left(s_{0}\right), x\left(s_{1}\right), \cdots, x\left(s_{r}\right)\right)^{2}-x(0)^{2} \\
& =-x(0)^{2}\left[1-\lambda^{2}\left(\frac{\left|g\left(x\left(s_{0}\right), x\left(s_{1}\right), \cdots, x\left(s_{r}\right)\right)\right|}{|x(0)|}\right)^{2}\right] \\
& \leq-x(0)^{2}\left[1-\lambda^{2} \alpha_{1}^{2}\right] \leq 0,
\end{aligned}
$$

we get

$$
\frac{1}{2}(x(T))^{2}-\frac{1}{2}(x(0))^{2}-\frac{1}{2} \sum_{k=1}^{m}\left[\left(x\left(t_{k}^{+}\right)\right)^{2}-\left(x\left(t_{k}^{-}\right)\right)^{2}\right] \leq \lambda M .
$$

Then

$$
\begin{aligned}
& \int_{0}^{T} h\left(s, x(s), x\left(\alpha_{1}(s)\right), \cdots, x\left(\alpha_{n}(s)\right)\right) x(s) d s+\int_{0}^{T} g_{0}(s, x(s)) x(s) d s \\
& +\sum_{i=1}^{n} \int_{0}^{T} g_{i}\left(s, x\left(\alpha_{i}(s)\right) x(s) d s+\int_{0}^{T} r(s) x(s) d s \leq M .\right.
\end{aligned}
$$


It follows from $(\mathbf{C})(\mathbf{i v})$ that

$$
\begin{aligned}
\beta \int_{0}^{T}|x(s)|^{\theta+1} d s \leq & \int_{0}^{T} h\left(s, x(s), x\left(\alpha_{1}(s)\right), \cdots, x\left(\alpha_{n}(s)\right)\right) x(s) d s \\
\leq & M-\int_{0}^{T} g_{0}(s, x(s)) x(s) d s-\sum_{i=1}^{n} \int_{0}^{1} g_{i}\left(s, x\left(\alpha_{i}(s)\right) x(s) d s\right. \\
& -\int_{0}^{T} r(s) x(s) d s \\
\leq & M+\sum_{i=0}^{n} \int_{0}^{T} \mid g_{i}\left(s, x\left(\alpha_{i}(s)\right)\left\|x(s)\left|d s+\int_{0}^{T}\right| r(s)\right\| x(s) \mid d s .\right.
\end{aligned}
$$

Since (2.1) holds, choose $\epsilon>0$ such that

$$
\left(r_{0}+\epsilon\right)+\sum_{k=1}^{n}\left(r_{k}+\epsilon\right)\left\|\beta_{k}^{\prime}\right\|^{\theta /(1+\theta)}<\beta .
$$

For such $\epsilon>0$, from $(\mathbf{C})(\mathbf{v})$, there exists a constant $\delta>0$ such that for every $i=0,1, \cdots, n$,

$$
\left|g_{i}(t, x)\right|<\left(r_{i}+\epsilon\right)|x|^{\theta} \text { uniformly for } t \in[0, T] \text { and }|x|>\delta .
$$

Let

$$
\begin{aligned}
\Delta_{1, i} & =\left\{t: t \in[0, T],\left|x\left(\alpha_{i}(t)\right)\right| \leq \delta\right\}, \quad i=1, \cdots, n, \\
\Delta_{2, i} & =\left\{t: t \in[0, T],\left|x\left(\alpha_{i}(t)\right)\right|>\delta\right\}, \quad i=1, \cdots, n, \\
g_{\delta, i} & =\max _{t \in[0, T],|x| \leq \delta}\left|g_{i}(t, x)\right|, \quad i=0,1, \cdots, n, \\
\Delta_{1} & =\{t \in[0, T],|x(t)| \leq \delta\}, \\
\Delta_{2} & =\{t \in[0, T],|x(t)|>\delta\} .
\end{aligned}
$$


Let $K=\max \left\{\|r\|, g_{\delta, i}: i=0,1, \cdots, n\right\}$. Then we get

$$
\begin{aligned}
& \beta \int_{0}^{T}|x(s)|^{\theta+1} d s \\
& \leq M+\sum_{i=0}^{n} \int_{\Delta_{2, i}} \mid g_{i}\left(s, x\left(\alpha_{i}(s)\right)|| x(s)\left|d s+\int_{0}^{T}\right| r(s)|| x(s) \mid d s\right. \\
& +\sum_{i=0}^{n} \int_{\Delta_{1, i}} \mid g_{i}\left(s, x\left(\alpha_{i}(s)\right)|| x(s) \mid d s\right. \\
& \leq\left(r_{0}+\epsilon\right) \int_{0}^{T}|x(s)|^{\theta+1} d s+\sum_{k=1}^{n}\left(r_{k}+\epsilon\right) \int_{0}^{T}\left|x\left(\alpha_{i}(s)\right)\right|^{\theta}|x(s)| d s \\
& +\int_{0}^{T}|r(s)||x(s)| d s+\sum_{k=0}^{n} g_{\delta, k} \int_{0}^{T} \mid x(s) d s \\
& \leq M+\left(r_{0}+\epsilon\right) \int_{0}^{T}|x(s)|^{\theta+1} d s \\
& +\sum_{k=1}^{n}\left(r_{k}+\epsilon\right)\left(\int_{0}^{T}\left|x\left(\alpha_{i}(s)\right)\right|^{\theta+1} d s\right)^{\frac{\theta}{1+\theta}}\left(\int_{0}^{T}|x(s)|^{\theta+1} d s\right)^{\frac{1}{1+\theta}} \\
& +K(n+2) T^{\frac{\theta}{1+\theta}}\left(\int_{0}^{T}|x(s)|^{\theta+1} d s\right)^{\frac{1}{1+\theta}} \\
& =M+\left(r_{0}+\epsilon\right) \int_{0}^{T}|x(s)|^{\theta+1} d s \\
& +\sum_{k=1}^{n}\left(r_{k}+\epsilon\right)\left(\int_{\alpha_{k}(0)}^{\alpha_{k}(T)}|x(u)|^{\theta+1}\left|\beta_{k}^{\prime}(u)\right| d u\right)^{\frac{\theta}{1+\theta}}\left(\int_{0}^{T}|x(s)|^{\theta+1} d s\right)^{\frac{1}{1+\theta}} \\
& +K(n+2) T^{\frac{\theta}{1+\theta}}\left(\int_{0}^{T}|x(s)|^{\theta+1} d s\right)^{\frac{1}{1+\theta}} \\
& \leq M+\left(r_{0}+\epsilon\right) \int_{0}^{T}|x(s)|^{\theta+1} d s \\
& +\sum_{k=1}^{n}\left(r_{k}+\epsilon\right)|| \beta_{k}^{\prime}||^{\frac{\theta}{1+\theta}}\left(\int_{0}^{T}|x(u)|^{1+\theta} d u\right)^{\frac{\theta}{1+\theta}}\left(\int_{0}^{T}|x(s)|^{\theta+1} d s\right)^{\frac{1}{1+\theta}} \\
& +K(n+2) T^{\frac{\theta}{1+\theta}}\left(\int_{0}^{T}|x(s)|^{\theta+1} d s\right)^{\frac{1}{1+\theta}} \\
& =M+\left(\left(r_{0}+\epsilon\right)+\sum_{k=1}^{n}\left(r_{k}+\epsilon\right)\left\|\beta_{k}^{\prime}\right\|^{\frac{\theta}{1+\theta}}\right) \int_{0}^{T}|x(s)|^{\theta+1} d s \\
& +K(n+2) T^{\frac{\theta}{1+\theta}}\left(\int_{0}^{T}|x(s)|^{\theta+1} d s\right)^{\frac{1}{1+\theta}} .
\end{aligned}
$$

That is

$$
\left(\beta-\left(r_{0}+\epsilon\right)-\sum_{k=1}^{n}\left(r_{k}+\epsilon\right)\left\|\beta_{k}^{\prime}\right\|^{\frac{\theta}{1+\theta}}\right) \int_{0}^{T}|x(s)|^{\theta+1} d s \leq M+K(n+2) T^{\frac{\theta}{1+\theta}}\left(\int_{0}^{T}|x(s)|^{\theta+1} d s\right)^{\frac{1}{1+\theta}} .
$$


It follows from (3.2) that there exists a constant $M_{1}>0$ such that $\int_{0}^{T}|x(s)|^{\theta+1} d s \leq M_{1}$. Hence there exists $\xi \in[0, T]$ such that $|x(\xi)| \leq\left(M_{1} / T\right)^{\frac{1}{\theta+1}}$.

Hence there exits $\xi \in[0, T]$ such that $|x(\xi)| \leq \max \left\{\delta^{\prime},\left(M_{1} / T\right)^{\frac{1}{\theta+1}}\right\}=: M^{\prime}$. Step 1 is complete.

Step 2. Prove that there exists a constant $M^{\prime \prime}>0$ such that $\|x\| \leq M^{\prime \prime}$.

If $t<\xi$, multiplying two sides of the first equation in (3.1) by $x(t)$, integrating it from $t$ to $\xi$, we get, using (A) and (C), similar to Step 1, that

$$
\begin{aligned}
& \frac{1}{2}(x(t))^{2}=\frac{1}{2}(x(\xi))^{2}-\frac{1}{2} \sum_{\xi \leq t_{k}<t}\left[\left(x\left(t_{k}^{+}\right)\right)^{2}-\left(x\left(t_{k}^{-}\right)\right)^{2}\right] \\
& -\lambda \int_{t}^{\xi} f\left(s, x(s), x\left(\alpha_{1}(s)\right), \cdots, x\left(\alpha_{n}(s)\right)\right) x(s) d s \\
& \leq M+\frac{1}{2} M^{\prime 2}-\lambda \int_{t}^{\xi} f\left(s, x(s), x\left(\alpha_{1}(s)\right), \cdots, x\left(\alpha_{n}(s)\right)\right) x(s) d s \\
& \leq M+\frac{1}{2} M^{\prime 2}-\lambda\left(\int_{t}^{\xi} h\left(s, x(s), x\left(\alpha_{1}(s)\right), \cdots, x\left(\alpha_{n}(s)\right)\right) x(s) d s\right. \\
& +\int_{t}^{\xi} g_{0}(s, x(s)) x(s) d s \\
& +\sum_{i=1}^{n} \int_{t}^{\xi} g_{i}\left(s, x\left(\alpha_{i}(s)\right) x(s) d s+\int_{t}^{\xi} r(s) x(s) d s\right) \\
& \leq M+\frac{1}{2} M^{\prime 2}-\beta \lambda \int_{t}^{\xi}|x(s)|^{\theta+1} d s-\lambda \int_{t}^{\xi} g_{0}(s, x(s)) x(s) d s \\
& -\lambda \sum_{i=1}^{n} \int_{t}^{\xi} g_{i}\left(s, x\left(\alpha_{i}(s)\right) x(s) d s-\lambda \int_{t}^{\xi} r(s) x(s) d s\right. \\
& \leq \quad M+\frac{1}{2} M^{\prime 2}+\sum_{i=0}^{n} \int_{0}^{T} \mid g_{i}\left(s, x\left(\alpha_{i}(s)\right)|| x(s)\left|d s+\int_{0}^{T}\right| r(s) \| x(s) \mid d s\right. \\
& \leq M+\frac{1}{2} M^{\prime 2}+\left(\left(r_{0}+\epsilon\right)+\sum_{k=1}^{n}\left(r_{k}+\epsilon\right)\left\|\beta_{k}^{\prime}\right\|^{\frac{\theta}{1+\theta}}\right) \int_{0}^{T}|x(s)|^{\theta+1} d s \\
& +(n+2) K T^{\frac{\theta}{1+\theta}}\left(\int_{0}^{T}|x(s)|^{\theta+1} d s\right)^{\frac{1}{\theta+1}} \\
& \leq M+\frac{1}{2} M^{\prime 2}+\left(\left(r_{0}+\epsilon\right)+\sum_{k=1}^{n}\left(r_{k}+\epsilon\right)\left\|\beta_{k}\right\|^{\theta /(1+\theta)}\right) M_{1} \\
& +(n+2) K T^{\frac{\theta}{1+\theta}} M_{1}^{\frac{1}{\theta+1}} \\
& =: M_{2} \text {. }
\end{aligned}
$$

Hence one sees that

$$
x^{2}(t) \leq 2 M_{2}=M_{3} \text { for } t \in[0, \xi]
$$


This implies $x^{2}(0) \leq M_{3}$. So

$$
\begin{aligned}
x^{2}(T) & =\lambda^{2} g\left(x\left(s_{0}\right), x\left(s_{1}\right), \cdots, x\left(s_{r}\right)\right)^{2} \\
& \leq \max \left\{\max _{\left|x_{0}\right| \leq \delta^{\prime}} g\left(x\left(s_{0}\right), x\left(s_{1}\right), \cdots, x\left(s_{r}\right)\right)^{2}, \max _{\delta^{\prime}<\left|x_{0}\right| \leq \sqrt{M_{3}}} \mid g\left(x\left(s_{0}\right), x\left(s_{1}\right), \cdots, x\left(s_{r}\right)\right)^{2}\right\} \\
& \leq \max \left\{\max _{\left|x_{0}\right| \leq \delta^{\prime}} g\left(x\left(s_{0}\right), x\left(s_{1}\right), \cdots, x\left(s_{r}\right)\right)^{2}, \max _{\delta^{\prime}<\left|x_{0}\right| \leq \sqrt{M_{3}}} \alpha_{1}^{2}\left|x\left(s_{0}\right)\right|^{2}\right\} \\
& \leq \max \left\{\max _{\left|x_{0}\right| \leq \delta^{\prime}} g\left(x\left(s_{0}\right), x\left(s_{1}\right), \cdots, x\left(s_{r}\right)\right)^{2}, \alpha_{1}^{2} M_{3}\right\} .
\end{aligned}
$$

It follows from (D) that there exists a constant $M_{4}>0$ such that $|x(T)| \leq M_{4}$. For $t \in[\xi, T]$, we have

$$
\begin{aligned}
\frac{1}{2}(x(t))^{2}= & \frac{1}{2}(x(T))^{2}-\frac{1}{2} \sum_{\xi \leq t_{k}<t}\left[\left(x\left(t_{k}^{+}\right)\right)^{2}-\left(x\left(t_{k}^{-}\right)\right)^{2}\right] \\
& -\lambda \int_{t}^{T} f\left(s, x(s), x\left(\alpha_{1}(s)\right), \cdots, x\left(\alpha_{n}(s)\right)\right) x(s) d s .
\end{aligned}
$$

Similar to above discussion, we get that there is $M_{5}>0$ so that $x^{2}(t) \leq M_{5}$ for $t \in[\xi, T]$. All above discussion implies that there is $M^{\prime \prime \prime}=\max \left\{M_{3}, M_{5}\right\}>0$ so that $|x(t)| \leq M^{\prime \prime \prime}$. Thus $\|x\| \leq M^{\prime \prime \prime}$.

It follows that $\Omega_{1}=\{x \in D(L): L x=\lambda N x$ for some $\lambda \in[0,1]\}$ is bounded.

Let $\Omega \supset \overline{\Omega_{1}}$ be an open bounded subset of $X$, it is easy to see that $L x \neq \lambda N x$ for all $x \in D(L) \cap \partial \Omega$ and $\lambda \in[0,1]$. It follows from Lemma 3.1 that equation $L x=N x$ has at least one solution $x \in \Omega$, then $x$ is a solution of IBVP(1.8). The proof is complete.

Remark 1. In Theorem 2.1, the assumption (D) may be changed into the following

$\left(\mathbf{D}^{\prime}\right)$. There exists constant $\delta^{\prime}>0$ such that

$$
\frac{\left|g\left(x_{0}, x_{1}, \cdots, x_{r}\right)\right|}{\left|x_{0}\right|} \leq 1 \text { for all }\left|x_{0}\right|>\delta^{\prime} \text { and }\left(x_{1}, \cdots, x_{r}\right) \in R^{r}
$$

Consider $\operatorname{BVP}(9)$, we define the linear operator $L_{1}: D\left(L_{1}\right) \subseteq X \rightarrow Y$ by

$$
L_{1} x(t)=\left(\begin{array}{c}
x^{\prime}(t) \\
\Delta x\left(t_{1}\right) \\
\cdot \\
\cdot \\
\cdot \\
\Delta x\left(t_{m}\right) \\
x(0)
\end{array}\right) \text { for } x \in D(L)
$$

where $D\left(L_{1}\right)=\left\{u \in X, u_{k} \in C^{1}\left(t_{k}, t_{k+1}\right), k=0,1, \cdots, m\right\}$ and the nonlinear operator $N: X \rightarrow Y$ is the same that for $\operatorname{IBVP}(8)$.

Proof of Theorem 2.2. Let $\lambda \in(0,1)$. Suppose $x$ is a solution of the system

$$
\left\{\begin{array}{l}
x^{\prime}(t)=\lambda f\left(t, x(t), x\left(\alpha_{1}(t)\right), \cdots, x\left(\alpha_{m}(t)\right)\right), \text { a.e. } t \in[0, T], \\
\Delta x\left(t_{k}\right)=\lambda I_{k}\left(x\left(t_{1}\right), \cdots, x\left(t_{m}\right)\right), k=1, \cdots, m, \\
x(0)=\lambda g\left(x\left(s_{0}\right), x\left(s_{1}\right), \cdots, x\left(s_{r}\right)\right) .
\end{array}\right.
$$

We divide the remainder of the proof into two steps.

Step 1. Prove that there exists $\xi \in[0, T]$ and a constant $M>0$ such that $|x(\xi)| \leq M$.

Since (D1) holds, we get that there exist constants $\delta^{\prime}>0$ and $\alpha_{1} \in[\alpha, 1)$ such that

$$
\frac{\left|g\left(x_{0}, x_{1}, \cdots, x_{r}\right)\right|}{\left|x_{r}\right|}<\alpha_{1} \text { for all }\left|x_{r}\right|>\delta^{\prime} \text { and }\left(x_{1}, \cdots, x_{r}\right) \in R^{r} .
$$


If $\left|x\left(s_{r}\right)\right|=|x(T)| \leq \delta^{\prime}$, then this step is completed with $\xi=T$ and $M=\delta^{\prime}$. If $|x(T)|>\delta^{\prime}$, then we do the following.

Multiplying two sides of the first equation in (3.4) by $x(t)$, integrating it from 0 to $T$, we get

$$
\begin{aligned}
& \frac{1}{2}(x(T))^{2}-\frac{1}{2}(x(0))^{2}-\frac{1}{2} \sum_{k=1}^{m}\left[\left(x\left(t_{k}^{+}\right)\right)^{2}-\left(x\left(t_{k}^{-}\right)\right)^{2}\right] \\
= & \lambda \int_{0}^{T} f\left(s, x(s), x\left(\alpha_{1}(s)\right), \cdots, x\left(\alpha_{n}(s)\right)\right) x(s) d s \\
= & \lambda\left(\int_{0}^{T} h\left(s, x(s), x\left(\alpha_{1}(s)\right), \cdots, x\left(\alpha_{n}(s)\right)\right) x(s) d s+\int_{0}^{T} g_{0}(s, x(s)) x(s) d s\right. \\
& +\sum_{i=1}^{n} \int_{0}^{T} g_{i}\left(s, x\left(\alpha_{i}(s)\right) x(s) d s+\int_{0}^{T} r(s) x(s) d s\right) .
\end{aligned}
$$

It follows from (A1) that

$$
\begin{aligned}
& \left(x\left(t_{k}^{+}\right)\right)^{2}-\left(x\left(t_{k}^{-}\right)\right)^{2}=\left(x\left(t_{k}^{+}\right)-x\left(t_{k}^{-}\right)\right)\left(x\left(t_{k}^{+}\right)+x\left(t_{k}^{-}\right)\right) \\
= & \Delta x\left(t_{k}^{-}\right)\left(2 x\left(t_{k}^{-}\right)+\Delta x\left(t_{k}^{-}\right)\right) \\
= & \lambda I_{k}\left(x\left(t_{1}\right), \cdots, x\left(t_{m}\right)\right)\left(2 x\left(t_{k}^{-}\right)+\lambda I_{k}\left(x\left(t_{1}\right), \cdots, x\left(t_{m}\right)\right)\right) \\
\leq & \lambda I_{k}\left(x\left(t_{1}\right), \cdots, x\left(t_{m}\right)\right)\left(2 x\left(t_{k}^{-}\right)+I_{k}\left(x\left(t_{1}\right), \cdots, x\left(t_{m}\right)\right)\right) \\
\leq & 2 \lambda \frac{M}{m} .
\end{aligned}
$$

Since

$$
\begin{aligned}
x(T)^{2}-x(0)^{2} & =[x(T)]^{2}-\lambda^{2} g\left(x\left(s_{0}\right), x\left(s_{1}\right), \cdots, x\left(s_{r}\right)\right)^{2} \\
& =x(T)^{2}\left[1-\lambda^{2}\left(\frac{\left|g\left(x\left(s_{0}\right), x\left(s_{1}\right), \cdots, x\left(s_{r}\right)\right)\right|}{|x(T)|}\right)^{2}\right] \\
& \geq x(T)^{2}\left[1-\lambda^{2} \alpha_{1}^{2}\right] \geq 0,
\end{aligned}
$$

we get

$$
\begin{aligned}
& \int_{0}^{T} h\left(s, x(s), x\left(\alpha_{1}(s)\right), \cdots, x\left(\alpha_{n}(s)\right)\right) x(s) d s+\int_{0}^{T} g_{0}(s, x(s)) x(s) d s \\
& +\sum_{i=1}^{n} \int_{0}^{T} g_{i}\left(s, x\left(\alpha_{i}(s)\right) x(s) d s+\int_{0}^{T} r(s) x(s) d s \geq M .\right.
\end{aligned}
$$

It follows from $\left(C_{1}\right)$ that

$$
\begin{aligned}
& \beta \int_{0}^{T}|x(s)|^{m+1} d s \\
\leq & M+\int_{0}^{T}\left|g_{0}(s, x(s))\right||x(s)| d s+\sum_{i=1}^{n} \int_{0}^{T} \mid g_{i}\left(s, x\left(\alpha_{i}(s)\right)|| x(s) \mid d s\right. \\
& +\int_{0}^{T}|r(s)||x(s)| d s .
\end{aligned}
$$

The remainder of the proof is similar to that of Theorem 2.1 and is omitted.

Remark 2. In Theorem 2.2, the assumption (D1) may be changed into the following

$\left(\mathbf{D} 1^{\prime}\right)$. There exist constants $\delta^{\prime}>0$ such that

$$
\frac{\left|g\left(x_{0}, x_{1}, \cdots, x_{r}\right)\right|}{\left|x_{r}\right|} \leq 1 \text { for all }\left|x_{r}\right|>\delta^{\prime} \text { and }\left(x_{0}, \cdots, x_{r-1}\right) \in R^{r}
$$




\section{Acknowledgements}

The authors thank the referee for the valuable comments and suggestions.

\section{References}

[1] A. Cabada, The monotone method for first order problems with linear and nonlinear boundary conditions, Appl. Math. Comput. 63 (1994), 163-186.

[2] D. Franco, J. J. Nieto, First order impulsive ordinary differential equations with anti-periodic and nonlinear boundary value conditions, Nonl. Anal. 42 (2000), 163-173.

[3] D. Franco, J. J. Nieto, A new maximum principle for impulsive first order problems, Internat. J. Theoret. Phys. 37 (1998), 1607-1616.

[4] R. Hakl, A. Lomtatidze, B. Puza, On a boundary value problem for first order scalar functional differential equations, Nonl. Anal. 53 (2003)391-405.

[5] Z. He, J. Yu, Periodic boundary value problems for first order impulsive ordinary differential equations, J. Math. Anal. Appl. 272 (2002), 67-78.

[6] D. Jiang, J. J. Nieto, W. Zuo, On monotone method for first order and second order periodic boundary value problems and periodic solutions of functional differential equations, J. Math. Anal. Appl. 289 (2004), 691-699.

[7] G. S. Ladde, V. Lakshmikantham, A. S. Vatsala, Monotone iterative techniques for nonlinear differential equations, Pitman Advanced Publishing Program, 1985.

[8] X. Li, X. Lin, D. Jiang, X. Zhang, Existence and multiplicity of positive periodic solutions to functional differential equations with impulse effects, Nonl. Anal., 62 (2005), 683-701.

[9] X. Liu, Nonlinear boundary value problems for first order impulsive integra-differential equations, Appl. Anal. 36 (1990), 119-130.

[10] J. J. Nieto, N. Alvarez-Noriega, Periodic boundary value problems for nonlinear first order ordinary differential equations, Acta Math. Hungar, 71(1996)49-58.

[11] J. J. Nieto, R. Rodriguez-Lopez, Periodic boundary value problem for non-Lipschitzian impulsive functional differential equations, J. Math. Anal. Appl. 318 (2006), 593-610.

[12] C. Pierson-Gorez, Impulsive differential equations of first order with periodic boundary conditions, Diff. Equs. Dyn. Systems, 11 (1993), 185-196.

[13] A. Cabada, The method of lower and upper solutions for second, third, fourth, and higher order boundary value problems, J. Math. Anal. Appl. 185 (1994), 302-320.

[14] A. Cabada, J. J. Nieto, D. Franco, S. I. Trofimchuk, A generalization of the monotone method for second order periodic boundary value problems with impulses at fixed points, Dynamics Contin. Discrete Impuls. System, 7 (2000), 145-158.

[15] A. S. Vatsala, Y. Sun, Periodic boundary value problems of impulsive differential equations, Appl. Anal. 44 (1992), 145-158.

[16] D. Franco, R.L. Pouso, Nonresonance conditions and extremal solutions for first order impulsive problems under weak assumptions, ANZIAM J. 44 (2003), 393-407.

[17] T. Jankowski, Existence of solutions of boundary value problems for differential equations with delayed arguments, J. Comput. Appl. Math. 156 (2003), 239-252.

[18] D. Franco, J. J. Nieto, D. O'Regan, Existence of solutions for first order ordinary differential equations with nonlinear boundary conditions, Appl. Math. Letters, 153 (2004), 793-802.

[19] T. Jankowski, Existence of solutions of differential equations with nonlinear multi-point boundary conditions, Comput. Math. Appl. 47 (2004), 1095-1103.

[20] J. J. Nieto, Impulsive resonance periodic problems of first order, Appl. Math. Letters, 15 (2002), 489-493.

[21] J. J. Nieto, Periodic boundary value problems for first order impulsive ordinary differential equations, Nonl. Anal. 51 (2002), 1223-1232.

[22] D. Franco, J. J. Nieto, Maximum principles for periodic impulsive first order problems, J. Comput. Appl. Math. 88 (1998), 144-159.

[23] Y. Liu, Further results on periodic boundary value problems for nonlinear first order impulsive functional differential equations, J. Math. Anal. Appl. 327 (2007), 435-452.

[24] Y. Liu, W. Ge, Stability theorems and existence results for periodic solutions of nonlinear impulsive delay differential equations with variable coefficients, Nonl. Anal. 57 (2004), 363-399.

[25] L. Kong, J. Sun, Nonlinear boundary value problem of first order impulsive functional differential equations, J. Math. Anal. Appl. 318 (2006), 726-741.

[26] E. Liz, Existence and approximation of solutions for impulsive first order problems with nonlinear boundary conditions, Nonl. Anal. 25 (1995), 1191-1198.

[27] X. Yang, J. Shen, Nonlinear boundary value problems for first order impulsive functional differential equations, Appl. Math. Comput. 189 (2007), 1943-1952.

[28] D. R. Smart, Fixed point theorems, Cambridge University Press, Cambridge, 1980. 
[29] S. Tang, L. Chen, Global attractivity in a "food-limited" population model with impulsive effects, J. Math. Anal. Appl. 292 (2004), 211-221.

[30] J. J. Nieto, Basic theory for nonresonance impulsive periodic problems of first order, J. Math. Anal. Appl. 205 (1997), 423-433.

[31] Z. Luo, Z. Jing, Periodic boundary value problem for first-order impulsive functional differential equations, Comput. Math. Appl. 55 (2008), 2094-2107.

[32] R. E. Gaines, J. L. Mawhin, Coincidence Degree and Nonlinear Differential Equations, Lecture Notes in Math., 568, Springer, Berlin, 1977.

[33] T. Jankowski, Ordinary differential equations with nonlinear boundary conditions of anti-periodic type, Comput. Math. Appl. 47 (2004), 1419-1428.

[34] J.J. Nieto, Differential inequalities for functional perturbations of first order ordinary differential equations, Appl. Math. Letters, 15 (2002), 173-179. 